\title{
MICROBIAL INVESTIGATION OF RECREATION EFFECTS ON WATER AND SOIL IN THE TATRA NATIONAL PARK
}

\author{
JANA JÚDOVÁ ${ }^{1}$, VERONIKA LACUŠOVÁ2, SIMONA KVASNOVÁ², JURAJ ŠVAJDA² \\ ${ }^{1}$ Alexander Dubček University of Trenčín, Faculty of Industrial Technologies in Púchov, I. Krasku 491/30, 02001 \\ Púchov, Slovak Republic, e-mail: jana.judova7@gmail.com \\ ${ }^{2}$ Matej Bel University, Faculty of Natural Sciences, Tajovského 40, 97401 Banská Bystrica, Slovak Republic
}

\begin{abstract}
Júdová J., Lacušová V., Kvasnová S., Švajda J.: Microbial investigation of recreation effects on water and soil in the Tatra National Park. Ekológia (Bratislava), Vol. 36, No. 3, p. 290-302, 2017.

Tatra National Park is considered as the most visited protected area in Slovakia. Striking a balance between the preservation of natural resources and opportunities for public recreation often forces responsible authorities to make compromises between visitation impacts and protection. In this case, the microbial investigation of recreation effects on water and soil in the Tatra National Park were studied.

The study areas were two valleys - Malá Studená, accessible by trail from south with higher human impact and visitation, including mountain huts Téryho and Zamkovského chata and Javorová, accessible from the northern part with a low number of visitors. Soil samples were taken from the main path, $30 \mathrm{~cm}$ away from it and water samples from or near the main path in both valleys. The selected colonies, after the cultivation on TSA medium were also analysed according to the dry and semi-extraction procedure of MALDI-TOF method. Most of the obtained strains are endospore forming, psychrotolerant species like Pseudomonas, Bacillus or Paenibacillus away of path, which corresponds with the climate and geographical conditions. But, the relatedness of soil sample strains in both valleys increases with rising altitude, with distance away from path; in contrast, the relatedness of water samples strains in both valleys increases with increasing distance sample areas from chalet and frequent visitors' places. Water and soil samples were processed for community level physiological profiling using Biolog EcoPlates. The obtained results of carbon source utilization abilities of bacterial communities in both valleys suggested lower diversity in Javorová Valley, which corresponds probably with less visitor intensity, with less anthropogenic impact as well as with less risk of xenobiotics presence in environment.
\end{abstract}

Key words: microorganisms, communities, impact, high mountain, visitors.

\section{Introduction}

Ecosystems, aquatic like terrestrial, have many parameters that interact to determine water quality. Some of these impact parameters are direct, occurring on or in the water, other impacts to water are indirect, characterized by inputs that originate from actions that occur on shore or in the watershed (Liddle, Scorgie, 1980; Newsome et al., 2013). Water impact reaches unacceptable levels commonly under the conditions of warm temperatures and low flow rate. Dissolved oxygen often reaches its lowest levels during warm summer 
evenings when water flow is also low. Warm temperatures tend to increase the growth of aquatic plants and bacteria, both of which are problems of warm-water systems. Water flow is related to dilution capacities, influencing the concentration of pollutants in water sources at any time. Restricted bays and inlets to lakes, as well as low-flowing springs and streams, often contain the highest bacterial counts and lowest oxygen supplies (Hammit et al., 2015). Precipitation patterns and the dilution capacity of a water system can greatly influence the degree of water recreation-caused impacts. However, the influence of storms is mixed. The rapid flushing rate of storms can help in removing suspended and dissolved nutrients from streams, but at the same time storms are a major agent in the flushing of nutrients and soil from disturbed watersheds into lakes and streams. Nutrient influx and coliform bacteria are sometimes most prevalent just after a storm. Potter et al. (1984) list natural sources to include precipitation, run-off, bottom, sediments, decomposing plankton, transient water flow, failing tree leaves, bedrock type and natural soils. Phosphate appears to be more of a nutrient input in wildland areas than nitrogen. A significant portion of the dissolved phosphorus is entering the eutrophic zone of the lake from apatite-rich rock, which has eroded following the destruction of ground cover as a result of human trampling (Hammit et al., 2015).

A major concern with recreational aquatic impacts involves the presence of pathogens and pollutants that directly influence human health. Pathogens are disease-causing organisms that are transmitted by the faeces of human and other warm-blooded animals. The presence of coliforms in a stream or lake usually indicates recent faecal pollution and the possible presence of enteric pathogens. Places where the water quality problems have been identified with recreational activities, most appear at those places where the use is concentrated and density dependent. Bacterial pollution resulting from recreation use appears to be more closely related to the total number of people visiting an area during a given seasonal period than to the total length of stay. Currently, little is known about the accumulation of bacterial organisms and nutrients in the bottom sediments of wildland water sources. Most studies of bacterial contamination examine only the surface waters when, in fact, the bottom sediments may contain the larger concentrations of organisms and nutrients. Brickler and Tunnicliff (1980) found FC (faecal coliforms) densities in bottom sediments to be significantly higher than in the surface water of rivers and tributaries in Arizona.

Water quality is a major concern, but not a frequent impact, in wildland recreation areas. Nutrient level, aquatic plant production and decomposition, and dissolved oxygen supply in aquatic ecosystems are all intricately related. The dominant source of bacterial contamination in drinking water has been wildlife, despite several studies assuming recreation to be the dominant impact.

Two hundred eighty-four isolates of enterococci from faeces of wild living chamois from alpine environments were tested for sensitivity to three antibiotics (Vandžurová et al., 2012). Low frequency of resistance was observed in the studied enterococcal populations (about 5\% for tetracycline and erythromycin, and $0 \%$ for ampicillin). In six animals, the population of enterococci lacked any detectable resistance. Data indicated that enterococcal population in the faeces of majority of studied animals did not encounter mobile genet- 
ic elements encoding antibiotic resistance, probably due to spatial separation and/or due to low exposure to the antibiotics. According to the obtained results, restriction-modification (R-M) systems do not represent an efficient barrier for antibiotic resistance spreading; enterococcal populations colonized by antibiotics resistance genes were also colonized by the R-M systems. Enterococci are gram-positive bacteria that normally colonize gastrointestinal tracts of warm-blooded animals and humans. Enterococci are infamous for their ability to acquire and transfer antibiotic resistance genes. These microorganisms inhabit different parts of the gastrointestinal tract, getting into contact with other bacteria, resulting in the transfer of genes through mechanisms such as conjugation, which occurs through the exchange of plasmids and conjugative transposons (Vandžurová et al., 2012).

In present work as well, the two valleys - Malá Studená (MSV) including mountain huts Téryho and Zamkovského chata, and Javorová (JV) - like the study areas were used. But in this case, the microbial investigation of recreation effects on water and soil in the Tatra National Park were studied.

\section{Material and methods}

\section{Description of the study area}

Malá Studená as well as Javorová valleys are situated in the eastern part of mountain High Tatras belonging to the western Carpathian mountain chain (Fig.1) (Koutná, Chovancová, 2010). Javorová valley is $8 \mathrm{~km}$ long and its feet are covered by coniferous forest. Circa to the start of upper forest border, the geology of the valley is diverse; there is alternation of marlstone and calcite, dark schist and muscovite schist, quartzite and dolomite, dark calcite and granodiorite. Here, Rendzina soil is the main soil type and the grey type of Rendzina soil and brown forest soil is found less frequently. Approximately from the beginning of the upper forest border, the diversity of geology starts to degrade. From this border, the geology consists of biotic granodiorite and silicate diorite, which represents the basis for mountainous podsol, and in subalpine and alpine level, are brown and grey subalpine soil. The path running in the Javorová Valley is at its start made mainly by trampled soil in combination with stones; the path in elevation from the second half of the valley is made by rocks. Nearly to the col and on it, the path is made of only big rocks and stones (Šoltés, 1985). The length of Malá Studená valley (Fig. 2) is $4.5 \mathrm{~km}$. Unlike Javorová valley, at the beginning of Malá Studená valley, a chalet was built called the Zamkovského chata. After the biggest slope made only by rocks, the second chalet in the valley named Téryho chata rises in $2015 \mathrm{~m}$ a.s.l. Near this lie five tarns - Five Spišské lakes. The highest placed tarn in High Tatras called Modré lake is situated under the highest point of both valleys - col Sedielko in $2372 \mathrm{~m}$ a.s.l. From a geological point of view, the path and its surroundings in Malá Studená valley is made of biotic granodiorites and siliceous diorites resistant to trampling, to water and wind erosion. Dominative soil type is mountainous podsol soil. This touristic path is under control and partly artificially made of big rocks and grit (Šoltés, 1985).

Sampling of soil and water was realized during September 2015, in two Tatra mountain valleys called Malá Studená and Javorová valleys. Altogether 17 samples were taken from both the valleys (possibly with consideration of taking three soil samples from the main path, three soil samples $30 \mathrm{~cm}$ away of the main path and three water samples from or near the main path in both valleys). Each sample was marked in GPS (Global Positioning System) and got a serial number depending on the order in the GPS device. In Javorová valley, the sampling started in spruce - mountain pine zone in the direction from village Tatranská Javorina to the col Sedielko with altogether six soil samples and three water samples. In Malá Studená valley, five samples of soil and three samples of water were accomplished in alignment with and on the touristic path from cottage Téryho chata to chalet Zamkovského chata (Table 1).

\section{Cultivation and Matrix assisted laser desorption/ionization, time of flight - MALDI-TOF}

Preparation of the suspensions of soil samples: $0.5 \mathrm{~g}$ of soil was homogenized in $2 \mathrm{ml}$ of sterile phosphate buffer (PBS). Water and soil suspension samples /50-100 $\mu \mathrm{l} /$ were placed on TSA (Trypton Soya agar, Oxoid) medium 


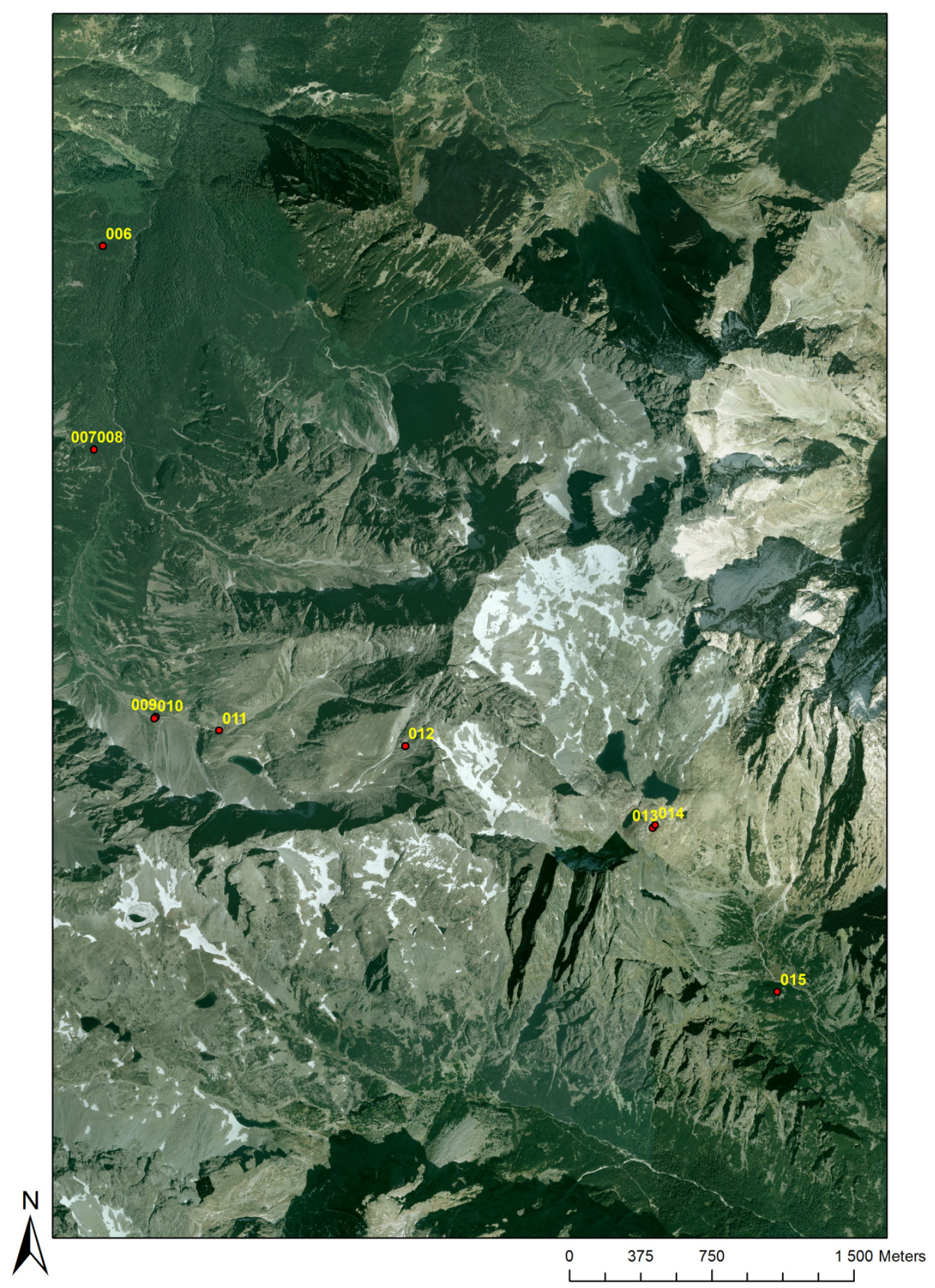

Fig. 1. Study areas (Malá Studená and Javorová valleys) with points highlighting samples. 


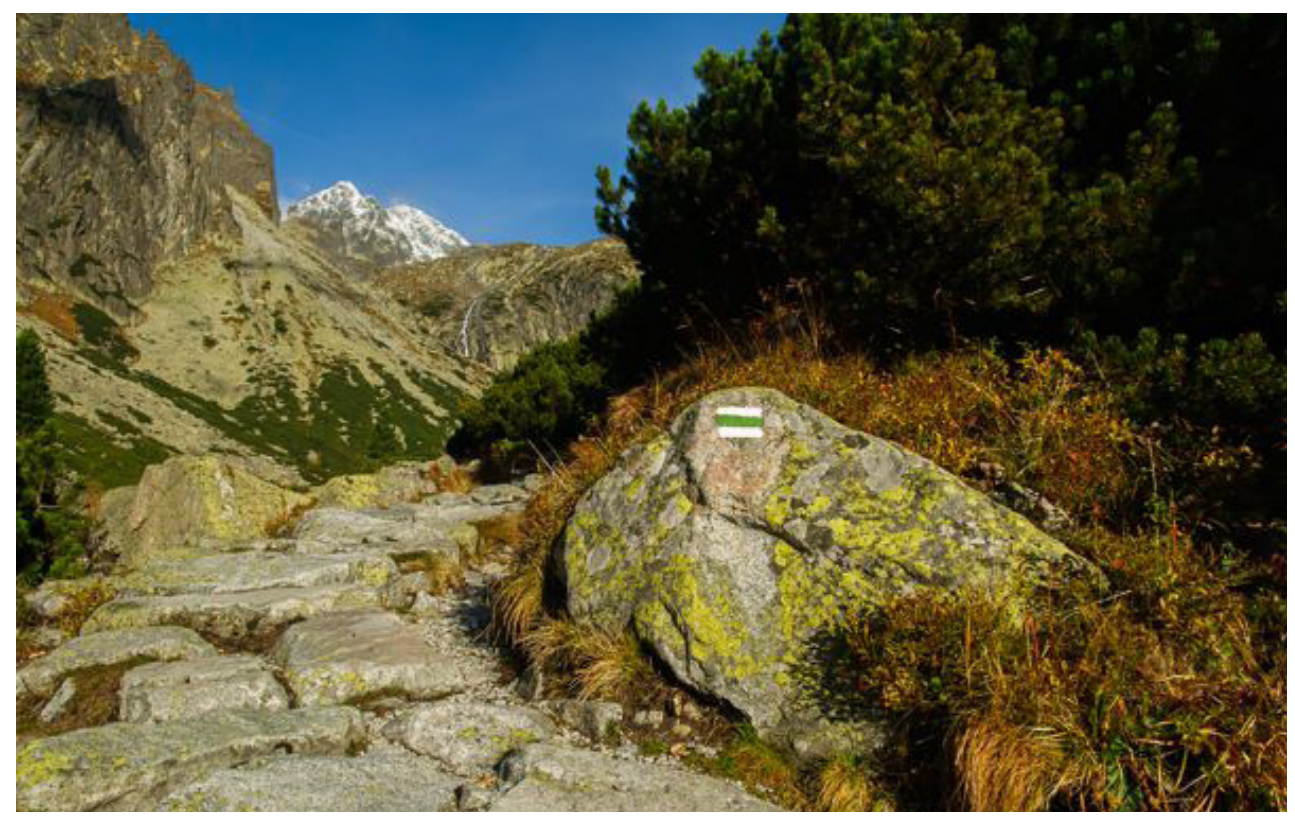

Fig. 2. Study area (Malá Studená valley).

and cultivated for 24 hours by $35^{\circ} \mathrm{C}$ (degrees Celsius) and then the concentration of colonies, colony forming units (cfu) were done (Table 1). The selected colonies were analysed according to the standard microbial protocols and dry and semi-extraction procedure of MALDI-TOF (Matrix assisted laser desorption/ionization, time of flight) method (Bruker protocols) (Table 2). Microbial communities' relationship dendrograms were generated according to the Maldi-Bruker software (Fig. 3).

\section{The Biolog EcoPlates}

Water and soil samples were processed for community level physiological profiling (CLPP) using Biolog EcoPlates (Garland, 1997). The Biolog EcoPlate contains 31 of the most useful carbon sources for soil community analysis. These 31 carbon sources are repeated 3 times to give the scientist more replicates of the data. Communities of organisms will give a characteristic reaction pattern called a metabolic fingerprint. Catabolism of each carbon substrate produced a proportional colour change response (from the colour of the inoculant to dark purple) due to the activity of the redox dye tetrazolium violet (present in all wells including blanks). The homogenized soil sample $(1 \mathrm{~g})$ was incubated in $99 \mathrm{ml}$ of sterile water for $20 \mathrm{~min}$ at $20^{\circ} \mathrm{C}$. Environmental samples $(150 \mu \mathrm{l})$ were then inoculated directly into Biolog MicroPlates (BioTech a.s.) either as aqueous samples and after suspension (soil) (Frac et al., 2012). The Biolog MicroPlates are incubated in the dark at $25^{\circ} \mathrm{C}$ and analysed at the defined time intervals over 2-5 days using the microplate reader by absorbance at $590 \mathrm{~nm}$ (Technical University, Zvolen, Slovakia). The rate of utilization was indicated by the reduction of tetrazolium, a redox indicator dye that changes from colourless into purple.

The changes in the pattern were compared via the Principle Components Analysis (PCA) of the average well colour development (AWCD) data (Firestone et al., 1997; Cartwright, 2015). Microbial response in each microplate that expressed the average well-colour development (AWCD) was determined as follows: AWCD = $\Sigma$ ODi/31 (Gomez et al., 2004), where ODi is the optical density value from each well, corrected subtracting the blank well (inoculated, but without a carbon source) values from each plate well (Table 3, Fig. 4). The changes observed in the fingerprint pattern provide useful data about the microbial population changes over time. 


\section{Results and discussion}

Sampling of soil and water was realized in two Tatra Mountain valleys called Malá Studená and Javorová valleys. Temperature of water was in the range from $4.9^{\circ} \mathrm{C}$ at beginning of the valley to $5.4^{\circ} \mathrm{C}$ in the middle, and to $9.2^{\circ} \mathrm{C}$ in the water in Spišské Mountain lake. The values of $\mathrm{pH}$ were $8-8.5$ in the Javorová valley and 7-7.5 in the Malá Studená valley (Table 1).

$\mathrm{T} \mathrm{a} \mathrm{b} \mathrm{l} \mathrm{e} \mathrm{1.} \mathrm{Description} \mathrm{of} \mathrm{sampling} \mathrm{areas} \mathrm{and} \mathrm{determination} \mathrm{of} \mathrm{cfu} \mathrm{(} \mathrm{cfu}$ - colony forming units, $10 \mathrm{~V}$ and $12 \mathrm{~V} \mathrm{sam-}$ ples - water were not available).

\begin{tabular}{|c|c|c|c|}
\hline \multicolumn{4}{|c|}{ Javorová valley } \\
\hline variable & GPS coordinates & Area description & $\mathrm{cfu} / \mathrm{ml}$ \\
\hline 006 Soil path / $06 \mathrm{P}$ & N49, 21608 E020, 14916 & $\begin{array}{l}\text { Start of valley; spruce - mountain pine and zone; } \\
1359 \text { m.a.s.l. }\end{array}$ & 850 \\
\hline 006 Water / 06V & N49, 21608 E020, 14916 & $\begin{array}{l}\text { Start of valley; rivulet crossing the path } \\
\text { spruce - mountain pine and zone; } 1359 \text { m.a.s.l. }\end{array}$ & 50 \\
\hline 007 Soil path / 07P & N49, 20642 E020, 15754 & Mountain pine zone, stone - soil; 1470 m.a.s.l. & 1200 \\
\hline 007 Water / 07V & N49, 20642 E020, 15754 & $\begin{array}{l}\text { Mountain pine zone rivulet; crossing the path; } \\
1470 \text { m.a.s.l. }\end{array}$ & 120 \\
\hline 008 Soil out of path / 08P & N49, 20641 E020, 15781 & $\begin{array}{l}30 \mathrm{~cm} \text { away from n.07P; } \\
\text { Mountain pine zone; } 1470 \text { m.a.s.l. }\end{array}$ & 1200 \\
\hline 009 Soil path / 09P & N49, 19392 E020,16346 & $\begin{array}{l}\text { Sparsely mountain pine; } \\
\text { rocky path; } 1729 \text { m.a.s.l. }\end{array}$ & 1000 \\
\hline 010 Soil out of path / 10P & N49, 19385 E020, 16335 & $\begin{array}{l}30 \mathrm{~cm} \text { away from n.09P } \\
\text { Sparsely mountain pine } 1730 \text { m.a.s.l. }\end{array}$ & 1300 \\
\hline 011 Water / 11V & N49, 19345 E020, 16808 & $\begin{array}{l}\text { po } 144 \text { m elevation from 09P, brooklet crossing } \\
\text { the path; } 1914 \text { m.a.s.l. }\end{array}$ & 260 \\
\hline 012 Soil / 12P & N49, 19326 E020, 18161 & Mountain col Sedielko; rocky path; 2373 m.a.s.l. & 1900 \\
\hline \multicolumn{4}{|c|}{ Malá Studená valley } \\
\hline sample no. & GPS coordinates & Area description & $\mathrm{cfu} / \mathrm{ml}$ \\
\hline 013 Soil path / 13P & N49, 19008 E020, 19984 & Near chalet Téryho chata; trampled soil; 2038 m.a.s.l. & 1200 \\
\hline 014 Soil out of path / 14P & N49, 19022 E020, 20003 & $30 \mathrm{~cm}$ away from 13P; 2007 m.a.s.l. & 1900 \\
\hline 014 Water / 14V & N49, 19022 E020, 20003 & chalet Téryho chata; last of 5 Spis Tarns; 2007 m.a.s.l. & 70 \\
\hline 015 Soil path / 15P/16P & N49, 18260 E020, 20956 & $\begin{array}{l}\text { Middle of chalet Téryho chata - chalet Zamkovs- } \\
\text { kého chata; mountain pine zone; } 1630 \text { m.a.s.l. }\end{array}$ & 1200 \\
\hline 015 Water / 15V & $\mathbf{N} 49,18260 \mathbf{E} 020,20956$ & $\begin{array}{l}\text { Middle of chalet Téryho chata - chalet Zamko- } \\
\text { vského chata mountain pine zone; brooklet } 1630 \\
\text { m.a.s.l. }\end{array}$ & 300 \\
\hline 017 Water / 17V & N49, 17368 E020, 21946 & $\begin{array}{l}\text { Puddle on the path near chalet Zamkovského } \\
\text { chata; spruce- mountain pine zone; } 1477 \text { m.a.s.l. }\end{array}$ & 320 \\
\hline 018 Soil path / 18P & $\mathbf{N} 49,17364 \mathbf{E} 020,21940$ & $\begin{array}{l}\text { the path near chalet Zamkovského chata; } \\
\text { spruce- mountain pine zone; } 1436 \text { m.a.s.l. }\end{array}$ & 1300 \\
\hline 019 Soil out of path/19P & N49, 17363 E020, 21937 & $\begin{array}{l}30 \mathrm{~cm} \text { away from 18P; near to chalet Zamkovského } \\
\text { chata; spruce - mountain pine zone; } 1464 \text { m.a.s.l. }\end{array}$ & 1300 \\
\hline
\end{tabular}


After the cultivation on TSA medium, selected colonies were analysed according standard microbial protocols (Table 1) and according dry and semi-extraction procedure of MALDITOF method (Bruker protocols) (Table 2).

T a b l e 2. MALDI-TOF analyses of selected bacterial strains from samples, different time of cultivations, 2a, 2b, 2c (Bruker Daltonik Biotyper Microflex LT).

a

\begin{tabular}{|c|c|c|c|c|c|}
\hline $\begin{array}{l}\text { Analyte } \\
\text { Name }\end{array}$ & $\begin{array}{l}\text { Analyte } \\
\text { ID }\end{array}$ & $\begin{array}{l}\text { Organism } \\
\text { (best match) }\end{array}$ & $\begin{array}{l}\text { Score } \\
\text { Value }\end{array}$ & $\begin{array}{l}\text { Organism } \\
\text { (second best match) }\end{array}$ & $\begin{array}{l}\text { Score } \\
\text { Value }\end{array}$ \\
\hline $\mathrm{G} 2(++)(\mathbf{B})$ & $11 \mathrm{~V}-1$ & Pseudomonas grimontii & 2.065 & Pseudomonas marginalis & 2.031 \\
\hline $\mathrm{G} 3(+)(\mathbf{B})$ & $11 \mathrm{~V}-2$ & Pseudomonas koreensis & 1.804 & Pseudomonas chlororaphis & 1.766 \\
\hline G5( + ) ( B ) & 09P-2 & Bacillus thuringiensis & 1.735 & not reliable identification & 1.265 \\
\hline $\mathrm{G} 6(+)(\mathbf{B})$ & $07 \mathrm{~V}-1$ & Pseudomonas chlororaphis & 1.952 & Pseudomonas thivervalensis & 1.798 \\
\hline $\mathrm{G} 8(+)(\mathbf{B})$ & 07P-1 & Pseudomonas jessenii & 1.724 & Pseudomonas fluorescens & 1.723 \\
\hline $\mathrm{G} 9(+)(\mathbf{B})$ & 07P-2 & Pseudomonas koreensis & 1.729 & Pseudomonas koreensis & 1.716 \\
\hline $\mathrm{G} 10(+)(\mathbf{B})$ & $08 \mathrm{P}-1$ & Paenibacillus amylolyticus & 1.988 & Paenibacillus amylolyticus & 1.971 \\
\hline $\mathrm{G} 12(+)(\mathbf{B})$ & $15 \mathrm{~V}-1$ & Pseudomonas extremorientalis & 1.841 & Pseudomonas umsongensis & 1.752 \\
\hline $\mathrm{H} 1(+)(\mathbf{B})$ & $15 \mathrm{~V}-2$ & Pseudomonas brassicacearum & 1.742 & Pseudomonas kilonensis & 1.735 \\
\hline $\mathrm{H} 2(+)(\mathbf{B})$ & $16 \mathrm{P}-1$ & Pseudomonas extremorientalis & 1.785 & Pseudomonas chlororaphis & 1.783 \\
\hline $\mathrm{H} 3(+)(\mathbf{B})$ & $16 \mathrm{P}-2$ & Pseudomonas kilonensis & 1.813 & Pseudomonas chlororaphis & 1.811 \\
\hline $\mathrm{H} 4(+)(\mathbf{C})$ & $14 \mathrm{P}-1$ & Bacillus weihenstephanensis & 1.996 & Pseudomonas kilonensis & 1.947 \\
\hline $\mathrm{H} 5(+)(\mathbf{B})$ & $14 \mathrm{P}-2$ & Paenibacillus amylolyticus & 1.998 & Paenibacillus amylolyticus & 1.991 \\
\hline $\mathrm{H} 7(+)(\mathbf{B})$ & $12 \mathrm{P}-2$ & Pseudomonas brassicacearum & 1.825 & Pseudomonas thivervalensis & 1.743 \\
\hline $\mathrm{H} 8(+)(\mathbf{B})$ & $12 \mathrm{P}-3$ & Pseudomonas thivervalensis & 1.865 & Pseudomonas rhodesiae & 1.745 \\
\hline $\mathrm{H} 12(++)(\mathbf{A})$ & $13 \mathrm{P}-2$ & Paenibacillus amylolyticus & 2.125 & Paenibacillus amylolyticus & 2.078 \\
\hline
\end{tabular}
b

\begin{tabular}{|c|c|c|c|c|c|}
\hline $\begin{array}{l}\text { Analyte } \\
\text { Name }\end{array}$ & $\begin{array}{l}\text { Analyte } \\
\text { ID }\end{array}$ & $\begin{array}{l}\text { Organism } \\
\text { (best match) }\end{array}$ & $\begin{array}{l}\text { Score } \\
\text { Value }\end{array}$ & $\begin{array}{l}\text { Organism } \\
\text { (second best match) }\end{array}$ & $\begin{array}{l}\text { Score } \\
\text { Value }\end{array}$ \\
\hline $\mathrm{A} 5(+)(\mathbf{B})$ & 08P-2 & Pseudomonas brassicacearum & 1.767 & Pseudomonas thivervalensis & 1.751 \\
\hline $\mathrm{A} 7(++)(\mathrm{B})$ & BTS & Escherichia coli & 2.247 & Escherichia coli & 2.241 \\
\hline $\mathrm{A} 8(+)(\mathbf{B})$ & 08P-1 & Paenibacillus amylolyticus & 1.771 & not reliable identification & 1.614 \\
\hline $\mathrm{A} 9(+)(\mathrm{B})$ & 09P-1 & Bacillus pumilus & 1.995 & Bacillus pumilus & 1.9 \\
\hline $\mathrm{A} 10(+)(\mathrm{B})$ & 09P-2 & Bacillus thuringiensis & 1.814 & Bacillus cereus & 1.813 \\
\hline $\mathrm{A} 11(+)(\mathrm{B})$ & $12 \mathrm{P}-1$ & Bacillus subtilis & 1.829 & Bacillus subtilis & 1.737 \\
\hline $\mathrm{B} 1(++)(\mathrm{A})$ & $12 \mathrm{P}-4$ & Stenotrophomonas rhizophila & 2.107 & not reliable identification & 1.273 \\
\hline $\mathrm{B} 2(+)(\mathrm{B})$ & $16 \mathrm{P}-1$ & Pseudomonas thivervalensis & 1.712 & not reliable identification & 1.588 \\
\hline $\mathrm{B} 5(+)(\mathrm{B})$ & $14 \mathrm{P}-2$ & Pseudomonas marginalis & 1.98 & Pseudomonas proteolytica & 1.833 \\
\hline $\mathrm{B} 7(+)(\mathrm{B})$ & $17 \mathrm{~V}-1$ & Pseudomonas chlororaphis & 1.942 & Pseudomonas corrugata & 1.878 \\
\hline $\mathrm{B} 8(+)(\mathbf{B})$ & $17 \mathrm{~V}-2$ & Serratia grimesii & 1.908 & Serratia liquefaciens & 1.793 \\
\hline $\mathrm{B} 9(+)(\mathrm{B})$ & $18 \mathrm{P}-1$ & Pseudomonas thivervalensis & 1.843 & Pseudomonas rhodesiae & 1.813 \\
\hline $\mathrm{B} 10(+)(\mathrm{B})$ & 19P-1 & Pseudomonas koreensis & 1.746 & not reliable identification & 1.692 \\
\hline B11( + ) ( B ) & $10 \mathrm{P}-1$ & Pseudomonas libanensis & 1.785 & Pseudomonas mandelii & 1.745 \\
\hline $\mathrm{C} 1(+)(\mathbf{B})$ & $06 \mathrm{~V}-2$ & Bacillus thuringiensis & 1.806 & Bacillus mycoides & 1.781 \\
\hline $\mathrm{C} 3(+)(\mathbf{B})$ & $06 \mathrm{~V}-3$ & Pseudomonas fragi & 1.848 & Pseudomonas rhodesiae & 1.733 \\
\hline
\end{tabular}




\begin{tabular}{|c|c|c|c|c|c|}
\hline $\begin{array}{l}\text { Analyte } \\
\text { Name }\end{array}$ & $\begin{array}{l}\text { Analyte } \\
\text { ID }\end{array}$ & $\begin{array}{l}\text { Organism } \\
\text { (best match) }\end{array}$ & $\begin{array}{l}\text { Score } \\
\text { Value }\end{array}$ & $\begin{array}{l}\text { Organism } \\
\text { (second best match) }\end{array}$ & $\begin{array}{l}\text { Score } \\
\text { Value }\end{array}$ \\
\hline $\mathrm{A} 3(+)(\mathbf{B})$ & $10 \mathrm{P}-2$ & Pseudomonas fluorescens & 1.907 & Pseudomonas antarctica & 1.902 \\
\hline $\mathrm{A} 4(+)(\mathbf{B})$ & $13 \mathrm{P}-3$ & Solibacillus silvestris & 1.757 & not reliable identification & 1.367 \\
\hline $\mathrm{A} 5(+)(\mathrm{B})$ & $11 \mathrm{~V}-2$ & Serratia liquefaciens & 1.802 & Serratia proteamaculans & 1.797 \\
\hline $\mathrm{A} 7(+)(\mathbf{B})$ & $16 \mathrm{P}-2$ & Pseudomonas brassicacearum & 1.888 & Pseudomonas frederiksbergensis & 1.848 \\
\hline $\mathrm{A} 8(+)(\mathrm{B})$ & $15 \mathrm{~V}-1$ & Pseudomonas extremorientalis & 1.878 & Pseudomonas chlororaphis & 1.802 \\
\hline $\mathrm{A} 10(+)(\mathrm{B})$ & 06P-1 & Pseudomonas fragi & 1.839 & not reliable identification & 1.521 \\
\hline $\mathrm{A} 12(+)(\mathbf{B})$ & $06 \mathrm{~V}-4$ & Bacillus weihenstephanensis & 1.949 & Bacillus cereus & 1.92 \\
\hline $\mathrm{B} 1(+)(\mathrm{B})$ & $13 \mathrm{P}-1$ & Pseudomonas fluorescens & 1.976 & Pseudomonas antarctica & 1.974 \\
\hline $\mathrm{B} 2(++)(\mathrm{B})$ & $13 \mathrm{P}-2$ & Pseudomonas fluorescens & 2.107 & Pseudomonas antarctica & 2.037 \\
\hline $\mathrm{B} 4(++)(\mathrm{A})$ & $14 \mathrm{~V}-2$ & Bacillus megaterium & 2.152 & not reliable identification & 1.416 \\
\hline $\mathrm{B} 5(+)(\mathrm{B})$ & $18 \mathrm{P}-2$ & Pseudomonas libanensis & 1.98 & Pseudomonas synxantha & 1.9 \\
\hline $\mathrm{B} 8(+)(\mathrm{B})$ & 19P-1 & Pseudomonas koreensis & 1.817 & Pseudomonas koreensis & 1.815 \\
\hline $\mathrm{C} 2(+)(\mathbf{B})$ & 08P-3 & Pseudomonas rhodesiae & 1.74 & not reliable identification & 1.681 \\
\hline $\mathrm{C} 3(+)(\mathbf{B})$ & 09P-1 & Pseudomonas thivervalensis & 1.893 & Pseudomonas brassicacearum & 1.822 \\
\hline $\mathrm{C} 5(+)(\mathbf{B})$ & 12P-1 & Pseudomonas brassicacearum & 1.804 & Pseudomonas thivervalensis & 1.76 \\
\hline $\mathrm{C} 8(+)(\mathbf{B})$ & $16 \mathrm{P}-1$ & Pseudomonas thivervalensis & 1.873 & Pseudomonas brassicacearum & 1.834 \\
\hline $\mathrm{C} 9(+)(\mathbf{B})$ & $16 \mathrm{P}-3$ & Pseudomonas frederiksbergensis & 1.766 & Pseudomonas kilonensis & 1.726 \\
\hline $\mathrm{C} 11(+)(\mathbf{B})$ & $14 \mathrm{P}-3$ & Pseudomonas antarctica & 1.906 & Pseudomonas fluorescens & 1.778 \\
\hline $\mathrm{C} 12(++)(\mathrm{B})$ & $13 \mathrm{P} 1-2$ & Pseudomonas fluorescens & 2.127 & Pseudomonas antarctica & 2.069 \\
\hline
\end{tabular}

Notes: Meaning of score values

\begin{tabular}{|l|l|c|c|}
\hline Range & Description & Symbols & Colour \\
\hline $2.300 \ldots \mathbf{3 . 0 0 0}$ & highly probable species identification & $(+++)$ & Green \\
\hline $\mathbf{2 . 0 0 0} \ldots \mathbf{2 . 2 9 9}$ & secure genus identification, probable species identification & $(++)$ & Green \\
\hline $1.700 \ldots 1.999$ & probable genus identification & $(+)$ & Yellow \\
\hline $\mathbf{0 . 0 0 0} \ldots \mathbf{1 . 6 9 9}$ & not reliable identification & $(-)$ & Red \\
\hline
\end{tabular}

Predictably, cfu of water samples were in range 50 (start of Javorová valley) - 300 cfu (middle of chalet Téryho chata - chalet Zamkovského chata, Malá Studená valley), but cfu of soil samples were in range 850 (start of Javorová valley) - $1900 \mathrm{cfu}$ (30 cm away from 13P - Malá Studená valley, near Téryho chata chalet, and mountain col Sedielko; rocky path; 2373 m.a.s.l. Javorová valley). Not only quantitative, but also qualitative microbial analyses of soil and water samples showed differences between areas, probably via different geological, natural and climate conditions and via impact of visitation as well. In the sample of mountain col Sedielko, Javorová valley, at rocky path, for example, strains like Pseudomonas brassicacearum, P. thivervalensis, Bacillus subtilis, Stenotrophomonas rhizophila were identified. In contrast, in sample near Téryho chata chalet (trampled soil; 2038 m.a.s.l. Malá Studená valley), strains like Pseudomonas fluorescens, Paenibacillus amylolyticus, Solibacillus silvestris were identified. 
Pseudomonas species are frequently isolated from different ecological niches, where some may act as pathogens of animals, plants or fungi and some are beneficial free-living soil bacteria referred to as plant-growth-promoting rhizobacteria. They are also frequently isolated from polluted sites containing high levels of pollutants such as heavy metals (Achouak, et al., 2000).

Stenotrophomonas species have an important ecological role in the element cycle in nature (Ikemoto et al., 1980). Growth takes place at $4-37^{\circ} \mathrm{C}$. Resistant to many antibiotics, such as penicillin, tobramycin, imipenem and ceftazidime, but susceptible to chloramphenicol, kanamycin and trimethoprim, sulfamethoxazole. Strains were plant-associated and isolated from the rhizosphere of oilseed rape and from the rhizosphere and geocaulsophere (tuber) of potato. Endophytic colonization was found (Wolf et al., 2002). Pseudomonas fluorescens: it is an obligate aerobe, with an extremely versatile metabolism that can be found in the soil and in water, and is non-pathogenic (Frank, 1997). Paenibacillus amylolyticus: facultatively anaerobic soil bacteria, endospores forming, non-pathogenic (Shida et al., 1997). Bacteria belonging to the genus Paenibacillus have been detected in a variety of environments, such as soil, water, rhizosphere, vegetable matter, forage and insect larvae, as well as clinical samples (Lal, Tabacchioni, 2009; McSpadden, 2004; Montes et al., 2004). A novel estuarine bacterial strain, Solibacillus silvestris AM1, produces an extracellular, thermostable and fibrous, glycoprotein bioemulsifier (BE-AM1). Cell-bound BE-AM1 production by $S$. silvestris AM1 during the mid-logarithmic phase of growth coincided with a decrease in the cell surface hydrophobicity, and an increase in the cell autoaggregation and biofilm formation. Markande and Nerurkar (2016) study has revealed that the BE-AM1, a bacterial bioemulsifier, is a functional amyloid and plays a role in biofilm formation and cell surface modulation in S. silvestris AM1. Sample areas $30 \mathrm{~cm}$ away from path in Javorová valley contain strains like Paenibacillus amylolyticus, Pseudomonas brassicacearum and P. fluorescens; in Malá Studená valley, strains like $P$. antarctica - psychrophilic bacterium were found that were isolated from the cyanobacterial mat samples collected from various water bodies in Antarctica (Reddy et al., 2004). Near the start of the valley, genus like P. fragi, $P$. thivervalensis were found. According to dendrogram 2 (Fig. 3), the relatedness of soil sample strains in both valleys increases with a rising altitude, with distance away path; in contrast, according to the dendrogram 1 (Fig. 3), the relatedness of water sample strains in both valleys increases with increasing distance sample areas from chalet and frequent visitors' places.

In Javorová valley, the water samples were identified with, for example, P. fragi, Bacillus thuringiensis, Serratia liquefaciens and in Malá Studená valley, with Pseudomonas extremorientalis, Serratia grimesi /liquefaciens and Bacillus megaterium. Serratia liquefaciens - facultative anaerobes is commonly found on many plant species, and more specifically, in the rhizosphere, it is responsible for about $2 \%$ of nosocomial infections. They tend to colonize in respiratory and urinary tracts of adults, resistant to antibiotics (Stock et al., 2003).

Commonly, most of the obtained strains are endospore forming, psychrotolerant species, which correspond with climate, seasonal and geographical conditions, like Pseudomonas, Bacillus or Paenibacillus away from the path.

Microbial communities' relationships dendrograms were generated according to the Maldi-Bruker software (Fig. 3). 


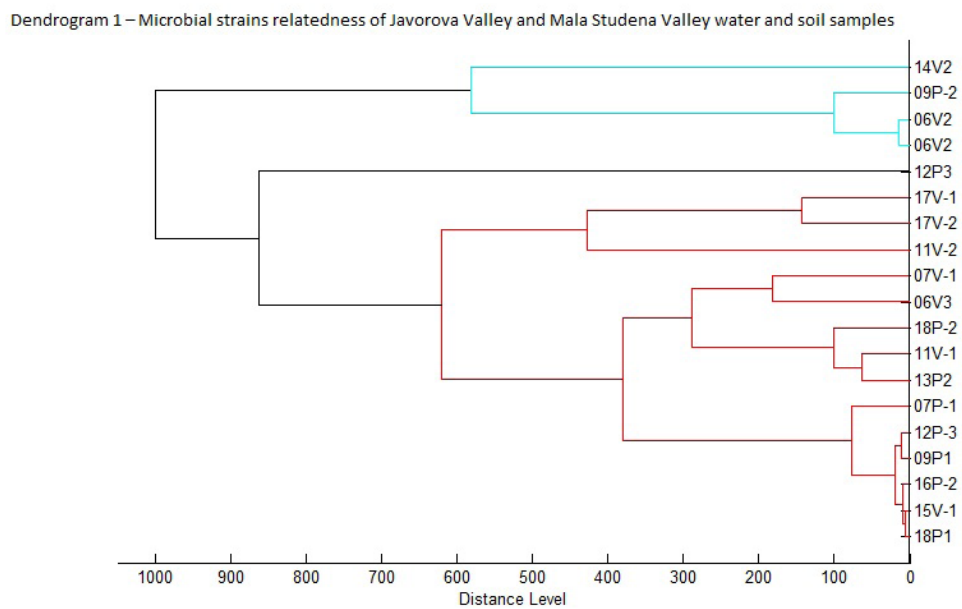

Dendrogram 2-Microbial strains relatedness of Javorova Valley and Mala Studena

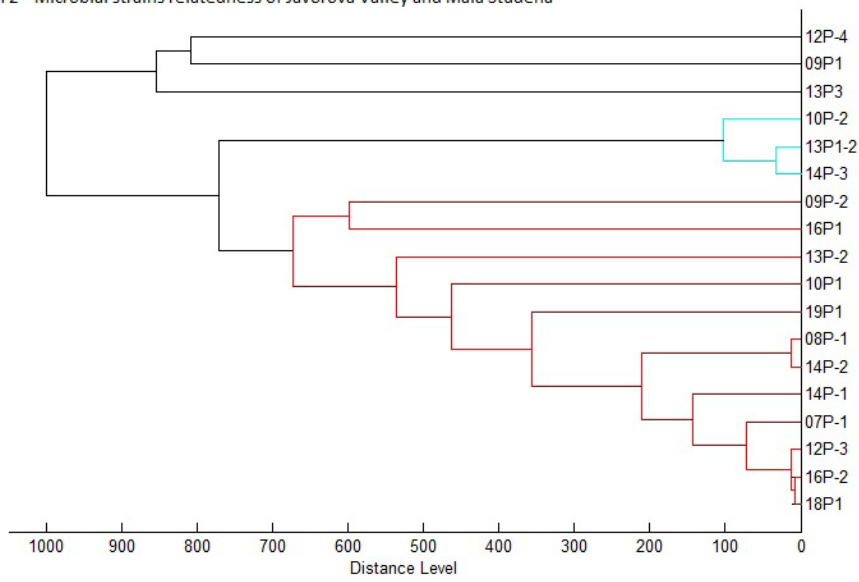

Fig. 3. Dendrograms: dendrogram 1- Microbial strains relatedness of Javorová valley and Malá Studená valley (water and soil samples), dendrogram 2 - Microbial strains relatedness of Javorová valley and Malá Studená valley (path soil samples).

\section{Biolog plates analyses}

The changes in the pattern, called a metabolic fingerprint, were compared via Principle Components Analysis (PCA) of average well colour development (AWCD) data (Table 3, Fig. 4).

Understanding how community processes affect the ecosystem processes is a central challenge in ecology, and microbial communities offer a potentially powerful forum for advancing this understanding (Garland, 1997). Also, active organisms, non-culturable using 
T a b l e 3. Average well colour development (AWCD).

\begin{tabular}{|l|c|c|c|c|c|c|}
\hline Sample & JD 006 & JD 009 & JD 012 & MSD 013 & MSD 016 & MSD 018 \\
\hline AWCD & 0,8224 & 0,616 & 0,3014 & 0,8075 & 0,8528 & 0,8192 \\
\hline
\end{tabular}

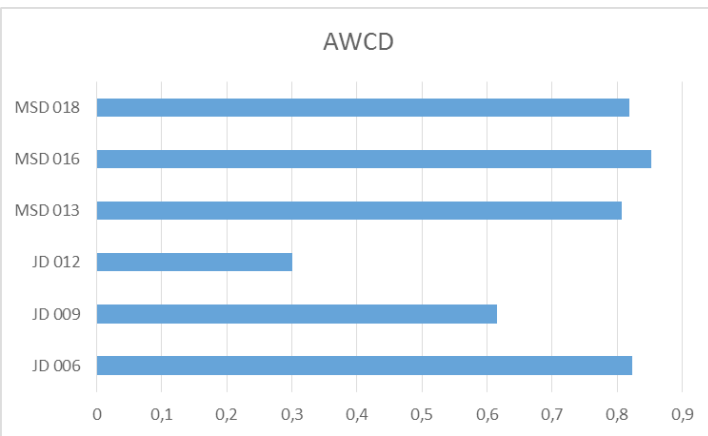

Fig. 4. Average well colour development (AWCD) data, MSD Malá Studená valley, JD - Javorová valley. solid agar, can respond in the CLPP. Organisms adapted for high nutrient concentration and rapid growth (like Pseudomonads) responds well on this assay (Garland, 1997). Measurements of metabolic traits through different carbon sources utilization are used like indicators. In Javorová valley, more colour intensity differences and a lot of colourless results were obtained as compared to Malá Studená valley (Fig. 4), especially in localities in high altitudes after the utilization of sources on Biolog plates. Therefore, the metabolic analyses of bacterial communities in the valley suggest lower diversity in Javorová valley, which corresponds probably with less visitors' intensity, with less anthropogenic impact and with less risk of xenobiotics presence in the environment. The presence of allochthonous components in the environment changes the representation and diversity of microbial communities in areas, especially in high mountain conditions with a fragile balance.

\section{Conclusion}

Identification and comparison of microbial community strains in selected localities should be integrant of visitation impact evaluation in the high mountain environment. Microbial community analyses provide information about the presence of strange strains, also pathogens in these ecosystems, which can survive in spite of allochthonous conditions and some of them can adapt to these conditions. Changes in environment, in contrast, subsequently provide changes in microbial community structures. In our study, the metabolic analyses of bacterial communities in the valley suggested a lower diversity in Javorová valley, which corresponds probably with less intensity of visitors, with less anthropogenic impact as well as with less risk of xenobiotics presence in the environment. Commonly, the strains obtained the most are endospore forming, psychrotolerant species, which correspond with climatic, seasonal and geographical conditions, like Pseudomonas, Bacillus or Paenibacillus away of path. But, the relatedness of soil sample strains in both valleys is increasing with rising altitude, with distance away path; in contrast, according to the dendrograms, the relatedness of water sample strains in both valleys increases with increasing distance sample areas from chalet and frequent visitors' places. The irreversible changes can be equilibrium of different 
symbiotic and commensal relations of water and soil environments, as well as of rhizosphere, fauna and flora. It also confirms studies about antibiotic resistance in faecal enterococci of chamois (Rupicapra rupicapra Linnaeus, 1758).

\section{Acknowledgements}

This study was supported by project 'Tourist visitation as a factor influencing diversity of organism in protected area' (code VEGA 1/0411/14) and by project 'Restoration and building of infrastructure for ecological and environmental research at Matej Bel University in Banská Bystrica' (code ITMS 26210120024). The support of research activities in Slovakia/Project is the co-financing from EU resources. Authors would like to thank Tomáš Lepeška for help with Figure 1 (map) production.

\section{References}

Achouak, W., Sutra, L., Heulin, T., Meyer, J.M., Fromin, N., Degraeve, S., Christen, R. \& Gardan L. (2000). Pseudomonas brassicacearum sp. nov. and Pseudomonas thivervalensis sp. nov., two root-associated bacteria isolated from Brassica napus and Arabidopsis thaliana. Int. J. Syst. Evol. Microbiol., 50, 9-18. DOI: 10.1099/0020771350-1-9.

Brickler, S.K. \& Tunnicliff B. (1980). Water quality analyses of the Colorado River Corridor of Grand Canyon. College of Agriculture Paper 350. Tucson: University of Arizona.

Cartwright, J.M. (2015). Average Well Color Development (AWCD) data based on Community Level Physiological Profiling (CLPP) of soil samples from 120 point locations within limestone cedar glades at Stones River National Battlefield near Murfreesboro, Tennessee: U.S. Geological Survey data release. DOI: 10.5066/F7NV9G9C.

Firestone, M., Balser, T. \& Herman D. (1997). Defining soil quality in terms of microbial community structure. Annual Reports of Research Projects. Berkeley: University of California.

Frac, M., Oszust, K. \& Lipiec J. (2012). Community level physiological profiles (CLPP) characterization and microbial activity of soil amended with dairy sewage sludge. Sensors, 12, 3253-3268. DOI: 10.3390/s120303253.

Frank, J.F. (1997). Milk and dairy products. In M.P. Doyle, L.R. Beuchat \& T.J. Montville (Eds.), Food microbiology, fundamentals and frontiers (p. 101). Washington: ASM Press.

Garland, J.L. (1997). Analysis and interpretation of community-level physiological profiles in microbial ecology. FEMS Microbiology Ecology, 24, p. 289-300. DOI: 10.1111/j.1574-6941.1997.tb00446.x.

Gomez, E., Garland, J. \& Conti M. (2004). Reproducibility in the response of soil bacterial community level physiological profiles from a land use intensification gradient. Appl. Soil Ecol. 26, 21-30. DOI: 10.1016/j.apsoil.2003.10.007.

Hammitt, W.E., Cole, D.N. \& Monz Ch. A. (2015). Wildland recreation: Ecology and management. Wiley Blackwell.

Ikemoto, S., Suzuki, K., Kaneko, T. \& Komagata K. (1980). Characterization of strains of Pseudomonas maltophilia which do not require methionine. Int. J. Syst. Evol. Microbiol., 30, 437-447. DOI: 10.1099/00207713-30-2-437.

Koutná, A. \& Chovancová B. (Eds.) (2010). Tatry - príroda. Praha: Baset.

Lal, S. \& Tabacchioni S. (2009). Ecology and biotechnological potential of Paenibacillus polymyxa: a minireview. Indian Journal of Microbiology, 49, 2-10. DOI: 10.1007/s12088-009-0008-y.

Liddle, M.J. \& Scorgie H.R.A. (1980). The effects of recreation on freshwater plants and animals: A review. Biol. Conserv., 17, 183-206. DOI: 10.1016/0006-3207(80)90055-5.

Markande, A.R. \& Nerurkar A.S. (2016). Bioemulsifier (BE-AM1) produced by Solibacillus silvestris AM1 is a functional amyloid that modulates bacterial cell-surface properties. Biofouling, 32(10), 1153-1162. DOI: 10.1080/08927014.2016.1232716.

McSpadden Gardener, B.B. (2004). Ecology of Bacillus and Paenibacillus spp. in agricultural systems. Phytopathology, 94, 252-1258. DOI: 10.1094/PHYTO.2004.94.11.1252.

Montes, M.J., Mercade, E., Bozal, N. \& Guinea J. (2004). Paenibacillus antarcticus sp. nov., a novel psychrotolerant organism from the Antarctic environment. Int. J. Syst. Evol. Microbiol., 54, 1521-1526. DOI: 10.1099/ ijs.0.63078-0.

Newsome, D., Moore, S. \& Dowling R. (2013). Natural area tourism: Ecology, impacts, and management. Bristol: Channel View Publications. 
Potter, L.D., Gosz, J.R. \& Carlson C.A. (1984). Forest recreational use, water, and aquatic life: An assessment of research results for land-use managers in the Southern CCRockies and High Plains. Eisenhower Consortium Bulletin No. 6. USDA Forest Service, Rocky Mountain Forest and Range Experiment Station.

Reddy, G.S., Matsumoto, G.I., Schumann, P., Stackebrandt, E. \& Shivaji S. (2004). “Psychrophilic pseudomonads from Antarctica: Pseudomonas antarctica sp. nov., Pseudomonas meridiana sp. nov. and Pseudomonas proteolytica sp. nov." Int. J. Syst. Bacteriol., 54, 713-719. DOI: 10.1099/ijs.0.02827-0.

Shida, O., Takagi, H., Kadowaki, K., Nakamura, L.K. \& Komagata K. (1997). Emended description of Paenibacillus amylolyticus and description of Paenibacillus illinoisensis sp. nov. and Paenibacillus chibensis sp. nov. Int. J. Syst. Evol. Microbiol., 47(2), 299-306. DOI: 10.1099/00207713-47-2-299.

Stock, I., Grueger, T. \& Wiedemann B. (2003). Natural antibiotic susceptibility of strains of Serratia marcescens and the S. liquefaciens complex: S. liquefaciens sensu stricto, S. proteamaculans and S. grimesii." Int. J. Antimicrob. Agents, 22(1), 35-47. DOI: 10.1016/S0924-8579(02)00163-2.

Šoltés, R. (1985). Únosná kapacita okolia turistických chodníkov vo Vysokých Tatrách z hladiska vegetačného krytu. Zborník prác o Tatranskom Národnom Parku, 26, 97-152.

Švajda, J. (2009). Contribution for improvement of visitor monitoring in the Tatra National Park. Eco.mont, 1(2), 13-18. DOI: 10.1553/ecomont2s13.

Vandžurová, A., Hrašková, I., Júdová, J., Javorský, P. \& Pristaš P. (2012). Antibiotic resistance and restriction endonucleases in fecal enterococci of chamois (Rupicapra rupicapra Linnaeus, 1758). Folia Microbiol., 57(4), 355-358. DOI: 10.1007/s12223-012-0141-6.

Wolf, A., Fritze, A., Hagemann, M. \& Berg G. (2002). Stenotrophomonas rhizophila sp. nov., a novel plant-associated bacterium with antifungal properties. Int. J. Syst. Evol. Microbiol., 5,2: 1937-1944. DOI: 10.1099/00207713-526-1937. 